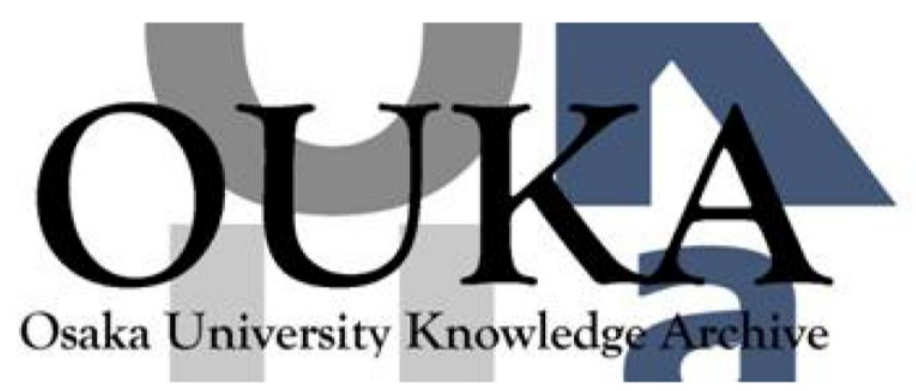

\begin{tabular}{|c|c|}
\hline Title & $\begin{array}{l}\text { Label-free observation of tissues by high-speed } \\
\text { stimulated Raman spectral microscopy and } \\
\text { independent component analysis }\end{array}$ \\
\hline Author (s) & $\begin{array}{l}\text { Ozeki, Yasuyuki; Otsuka, Yoichi; Sato, Shuya et } \\
\text { al. }\end{array}$ \\
\hline Citation & $\begin{array}{l}\text { Proceedings of SPIE - The International Society } \\
\text { for Optical Engineering. } 8588 \text { p. } 858806\end{array}$ \\
\hline Issue Date & $2013-02-22$ \\
\hline oaire:version & VoR \\
\hline URL & https://hdl. handle. net/11094/79144 \\
\hline rights & $\begin{array}{l}\text { Copyright } 2013 \text { Society of Photo Optical } \\
\text { Instrumentation Engineers (SPIE). One print or } \\
\text { electronic copy may be made for personal use } \\
\text { only. Systematic reproduction and distribution, } \\
\text { duplication of any material in this publication } \\
\text { for a fee or for commercial purposes, or } \\
\text { modification of the contents of the publication } \\
\text { are prohibited. }\end{array}$ \\
\hline Note & \\
\hline
\end{tabular}

Osaka University Knowledge Archive : OUKA

https://ir. Library. osaka-u. ac. jp/

0saka University 


\section{Label-free observation of tissues by high-speed stimulated Raman spectral microscopy and independent component analysis}

Ozeki, Yasuyuki, Otsuka, Yoichi, Sato, Shuya, Hashimoto, Hiroyuki, Umemura, Wataru, et al.

Yasuyuki Ozeki, Yoichi Otsuka, Shuya Sato, Hiroyuki Hashimoto, Wataru Umemura, Kazuhiko Sumimura, Norihiko Nishizawa, Kiichi Fukui, Kazuyoshi Itoh, "Label-free observation of tissues by high-speed stimulated Raman spectral microscopy and independent component analysis," Proc. SPIE 8588, Multiphoton Microscopy in the Biomedical Sciences XIII, 858806 (22 February 2013); doi: $10.1117 / 12.2002772$

SPIE. Event: SPIE BiOS, 2013, San Francisco, California, United States 


\title{
Label-free observation of tissues by high-speed stimulated Raman spectral microscopy and independent component analysis
}

\author{
Yasuyuki Ozeki*a,b, Yoichi Otsuka ${ }^{c}$, Shuya Sato ${ }^{c}$, Hiroyuki Hashimoto ${ }^{c}$, Wataru Umemura ${ }^{\mathrm{a}}$, \\ Kazuhiko Sumimura $^{\mathrm{a}}$, Norihiko Nishizawa ${ }^{\mathrm{d}}$, Kiichi Fukui ${ }^{\mathrm{a}}$ and Kazuyoshi Itoh $^{\mathrm{a}}$ \\ a Department of Advanced Science and Biotechnology, Graduate School of Engineering, \\ Osaka University, Suita, Osaka, Japan 565-0871; \\ b PRESTO, Japan Science and Technology Agency (JST), Kawaguchi, Saitama, Japan 332-0012; \\ ${ }^{\mathrm{c}}$ Canon Inc., Frontier Research Center, \\ 30-2 Shimomaruko 3-chome, Ota-ku, Tokyo 146-8501, Japan; \\ ${ }^{\mathrm{d}}$ Department of Electrical Engineering and Computer Science, Graduate School of Engineering, \\ Nagoya University, Nagoya, Japan 464-8603;
}

\begin{abstract}
We have developed a video-rate stimulated Raman scattering (SRS) microscope with frame-byframe wavenumber tunability. The system uses a 76-MHz picosecond Ti:sapphire laser and a subharmonically synchronized, $38-\mathrm{MHz} \mathrm{Yb}$ fiber laser. The $\mathrm{Yb}$ fiber laser pulses are spectrally sliced by a fast wavelength-tunable filter, which consists of a galvanometer scanner, a 4-f optical system and a reflective grating. The spectral resolution of the filter is $\sim 3 \mathrm{~cm}^{-1}$. The wavenumber was scanned from 2800 to $3100 \mathrm{~cm}^{-1}$ with an arbitrary waveform synchronized to the frame trigger. For imaging, we introduced a $8-\mathrm{kHz}$ resonant scanner and a galvanometer scanner. We were able to acquire SRS images of $500 \times 480$ pixels at a frame rate of 30.8 frames/s. Then these images were processed by principal component analysis followed by a modified algorithm of independent component analysis. This algorithm allows blind separation of constituents with overlapping Raman bands from SRS spectral images. The independent component (IC) spectra give spectroscopic information, and IC images can be used to produce pseudo-color images. We demonstrate various label-free imaging modalities such as 2D spectral imaging of the rat liver, two-color 3D imaging of a vessel in the rat liver, and spectral imaging of several sections of intestinal villi in the mouse. Various structures in the tissues such as lipid droplets, cytoplasm, fibrous texture, nucleus, and water-rich region were successfully visualized.
\end{abstract}

Keywords: stimulated Raman scattering (SRS) microscopy, spectral imaging, independent component analysis

\section{INTRODUCTION}

Recent development of stimulated Raman scattering (SRS) microscopy [1-3] offers unprecedented capability for label-free imaging such as high-speed imaging at up to the video rate [4] and chemical contrast based on vibrational spectroscopy without spectral distortion [1-3]. Fast imaging is especially important in biomedical imaging because it allows us to successively observe samples in different fields of view, and the images become less sensitive to artifacts due to the sample movement or degradation. In SRS microscopy, two-color laser pulses at the pump frequency $\omega_{\mathrm{p}}$ and the Stokes frequency $\omega_{\mathrm{s}}$ are used, and either the pump or Stokes beam is intensity-modulated beforehand. After these pulses are focused on the sample, the modulation transfer to the other beam due to SRS is measured by the lock-in detection technique. Several reports [1-13] have demonstrated 
the advantages of SRS in terms of sensitivity and chemical specificity over existing Raman microscopy techniques.

However, chemical specificity is still limited in the original implementation of SRS microscopy [1$3]$ because an SRS image reflects a single vibrational mode at $\omega_{p}-\omega_{s}$. This makes it difficult to specifically detect molecules with overlapping Raman bands. For further improving the specificity, tailored excitation SRS microscopy was demonstrated [14]. This technique uses narrowband Stokes pulses and spectrally tailored pump pulses to detect specific molecules. However, this technique requires a priori knowledge of the sample molecules. Similar limitations arise in multiplex detection techniques [15-16] although it allows us to detect several kinds of molecules simultaneously. Femtosecond SRS microscopy [17] can acquire Raman spectra with a spectrometer, but requires intense excitation pulses, which are not compatible with biological imaging. Another approach for pushing the chemical specificity is SRS spectral imaging [18-22], where SRS images at various vibrational frequencies are successively acquired. This may allow us to analyze vibrational spectrum so as to specifically detect molecules. However, in the previous reports on SRS spectral imaging, the acquisition time was several minutes or longer, limited by the tuning mechanisms or the microscope itself. Furthermore, it seems troublesome to analyze SRS spectral images, which contain tens of thousands of vibrational spectra. Here we introduce our recent development of a high-speed SRS spectral imaging system and a spectral analysis method for label-free imaging of tissues [23].

\section{SRS SPECTRAL MICROSCOPY AND INDEPENDENT COMPONENT ANALYSIS}

Figure 1a shows the schematic of the system. We synchronized two-color laser sources [24-25] equipped with a galvanometer-controlled wavelength scanner [21] with a tunability of $\sim 300 \mathrm{~cm}^{-1}$ and a spectral resolution of $\sim 3 \mathrm{~cm}^{-1}$ (Fig. 1b). By incorporating a resonant galvanometer scanner and a high-speed lock-in amplifier, we were able to acquire SRS images with $500 \times 480$ pixels at a frame rate of 30.8 frames/s while the wavelength scanner was controlled in a frame-by-frame manner.
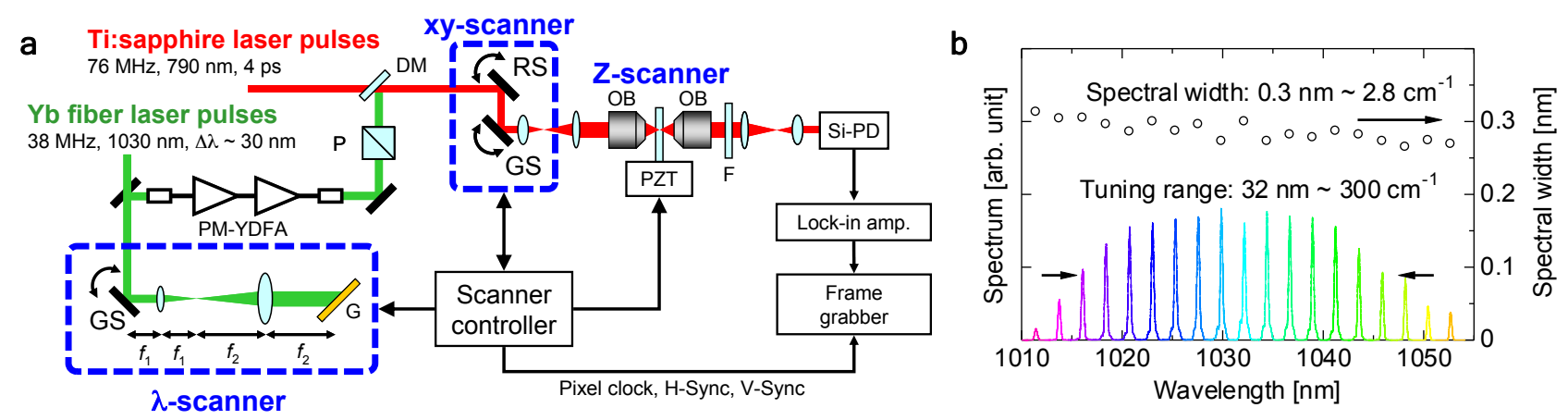

Fig. 1. High-speed SRS spectral microscopy [23]. a. Schematic of our experimental setup. Modulation transfer from $\mathrm{Yb}$ fiber laser (Stokes) pulses to Ti:sapphire laser (pump) pulses was detected. Images with $500 \times 480$ pixels are taken at 30.8 frames/s, while Stokes wavelength can be tuned by a galvanometer mirror in the wavelength scanner in a frame-by-frame manner. PM-YDFA: polarization maintaining Yb-doped fiber amplifier. GS: galvanometer scanner. RS: resonant galvanometer scanner. P: polarizer. OB: objective lens. PZT: piezoelectric transducer. b. Output spectra and spectral width of Stokes pulses with a wavelength tunability of $>300 \mathrm{~cm}^{-1}$. 
Figure 2 shows the results of spectral imaging of polymer beads. We observed $\sim 5-\mu$ m poly(methyl methacrylate) (PMMA) beads and 6- $\mu$ m polystyrene (PS) beads in water. We acquired 91 spectral images at wavenumbers ranging from 2800 to $3100 \mathrm{~cm}^{-1}$ within $3 \mathrm{~s}$. We can see that Fig. 2a-c have different contrast depending on the wavenumber. Figure $2 \mathrm{~d}$ shows the spectra taken at different locations indicated by the arrows in Fig. $2 b$ and 2c. Obviously, these spectra have different shape, and indicate the high signal-to-noise ratio of our imaging system.
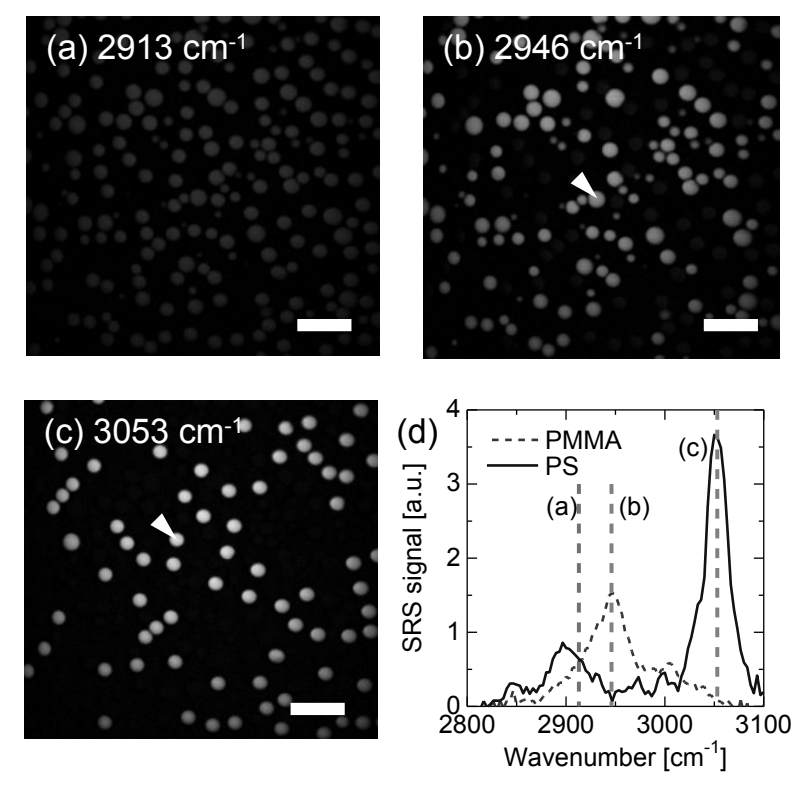

Fig. 2. Spectral imaging of poly(methyl methacrylate) (PMMA) and polystyrene (PS) beads. a-c: SRS images taken at 2913, 2946, and $3053 \mathrm{~cm}^{-1}$, respectively. d: SRS spectra taken at the locations of arrows in b and c. Scale bar: $20 \mu \mathrm{m}$.

The spectral data were analyzed by principal component analysis (PCA) followed by a modified version of independent component analysis (ICA) [26]. PCA allows one to extract characteristic features with a smaller number of dimensions. Then ICA is used for the blind separation of independent sources. Ordinary ICA assumes that the data given are linear combination of independent signal sources. Blind separation is possible through the iterative calculations, which look for the spectral bases whose linear projections maximize the difference of $4^{\text {th }}$-order moment (kurtosis) of probability density from that of Gaussian distribution because independent sources have maximum non-Gaussianity. Although ICA has been applied to the analysis of Raman spectral imaging [27], we found that modification is needed because the ordinary ICA assumes that the average values of sources and those of signals are zero, and the results of ICA tend to give bipolar values. In contrast, Raman spectra and Raman images always have positive values. To cope with this, we modified the ICA algorithm so that it maximizes the $3^{\text {rd }}$ order moment (skewness) of probability density. As a result, the modified ICA enables blind separation of positive sources and tends to give images with positive values [23].

Figure 3 demonstrates the source separation through the SRS spectral imaging and the modified ICA [23]. Figures $3 \mathrm{a}$ shows the spectral bases obtained by ICA. Through a simple inverse matrix 
calculation, we can calculate independent component (IC) spectra shown in Fig. 3b, which are in good agreement with those of PMMA and PS shown in Fig. 2d. From the IC spectra, we can speculate that the $1^{\text {st }}$ and $2^{\text {nd }}$ independent component (IC) images in Figs $3 \mathrm{c}$ and $3 \mathrm{~d}$ correspond to PMMA and PS, respectively. In this way, ICA allows blind source separation for SRS spectral images, and IC spectra can be used for the assignment of IC images.
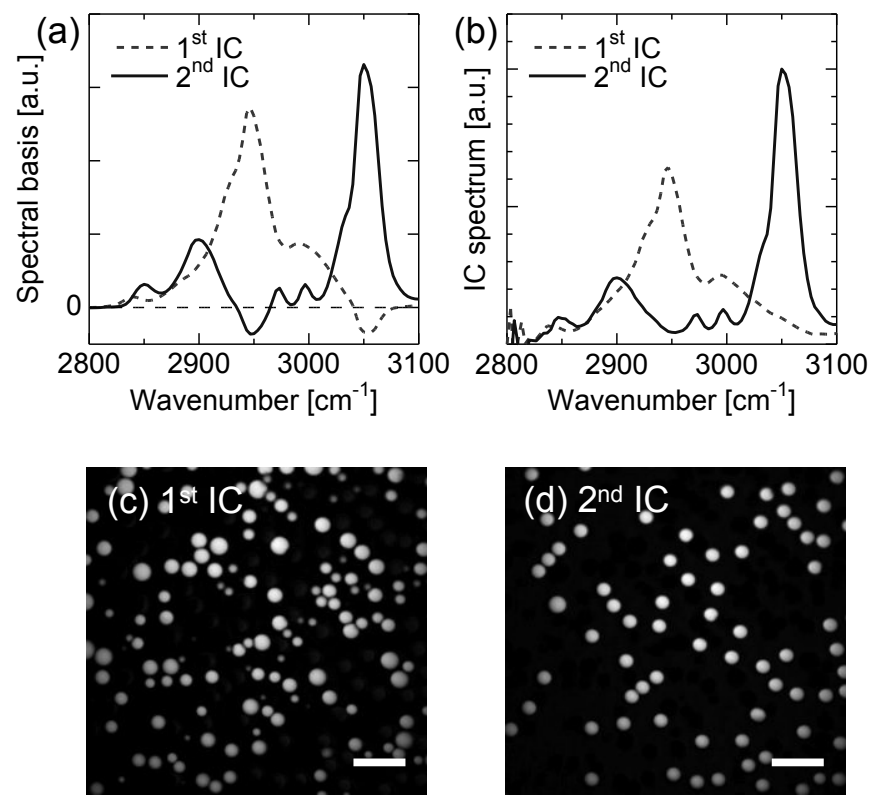

Fig. 3. Source separation by ICA. a. Spectral bases obtained by ICA. b. IC spectra. c-d. $1^{\text {st }}$ and $2^{\text {nd }}$ IC images, reflecting the distributions of PMMA and PS, respectively. Scale bar: $20 \mu \mathrm{m}$.

\section{TISSUE IMAGING}

Figure 4 shows the imaging results of the rat liver observed with the developed system. The tissue was cryo-sectioned into a nominal thickness of $100 \mu \mathrm{m}$, and preserved between cover glasses with phosphate buffered saline ( $\mathrm{pH7.4).} \mathrm{In} \mathrm{order} \mathrm{to} \mathrm{have} \mathrm{good} \mathrm{signal-to-noise} \mathrm{ratio,} \mathrm{we} \mathrm{repeated} 10$ times the acquisition of 91 spectral images at wavenumbers from 2800 to $3100 \mathrm{~cm}^{-1}$. Nevertheless, the total acquisition time is less than $30 \mathrm{~s}$. Figures $4 \mathrm{a}-\mathrm{c}$ show the $1^{\text {st }}, 2^{\text {nd }}$, and $3^{\text {rd }}$ IC images, respectively. These images correspond to the distributions of (a) lipids and cytoplasm, (b) water-rich regions, and (c) fibrous texture and nuclei, respectively. Their corresponding spectra shown in Fig. 4d indicate that the spectral difference is quite small. Al1 the IC spectra have $\mathrm{CH}_{3}$ stretching mode at $2930 \mathrm{~cm}^{-1}$, $\mathrm{CH}_{2}$ stretching vibration in $2850 \mathrm{~cm}^{-1}$, and the tails of $\mathrm{OH}$ stretching vibrations centered on $\sim 3400$ $\mathrm{cm}^{-1}$. However, their ratios of vibrational modes are different: The $1^{\text {st }}$ IC has prominent $\mathrm{CH}_{2}$ stretching mode, the $2^{\text {nd }} \mathrm{IC}$ is dominated by the $\mathrm{OH}$ stretching mode, and the $3^{\text {rd }} \mathrm{IC}$ has strong $\mathrm{CH}_{3}$ stretching mode. By combining these images and inverting the contrast [23], we could obtain a multicolor image shown in Fig. 4e. Various structures in the liver tissue such as lipid droplets (A), cytoplasm (B), fibrous texture (C), nucleus (D), and water-rich region (E) can be seen with different pseudo-colors, and their morphological shapes and locations are clearly visualized, which would be useful for pathological diagnosis. It is interesting to compare the IC spectra with the original SRS 
spectra shown in Fig. 4f. Obviously, IC spectra have much higher signal-to-noise ratio because ICA can extract spectral features from enormous number of pixels in a statistical manner.
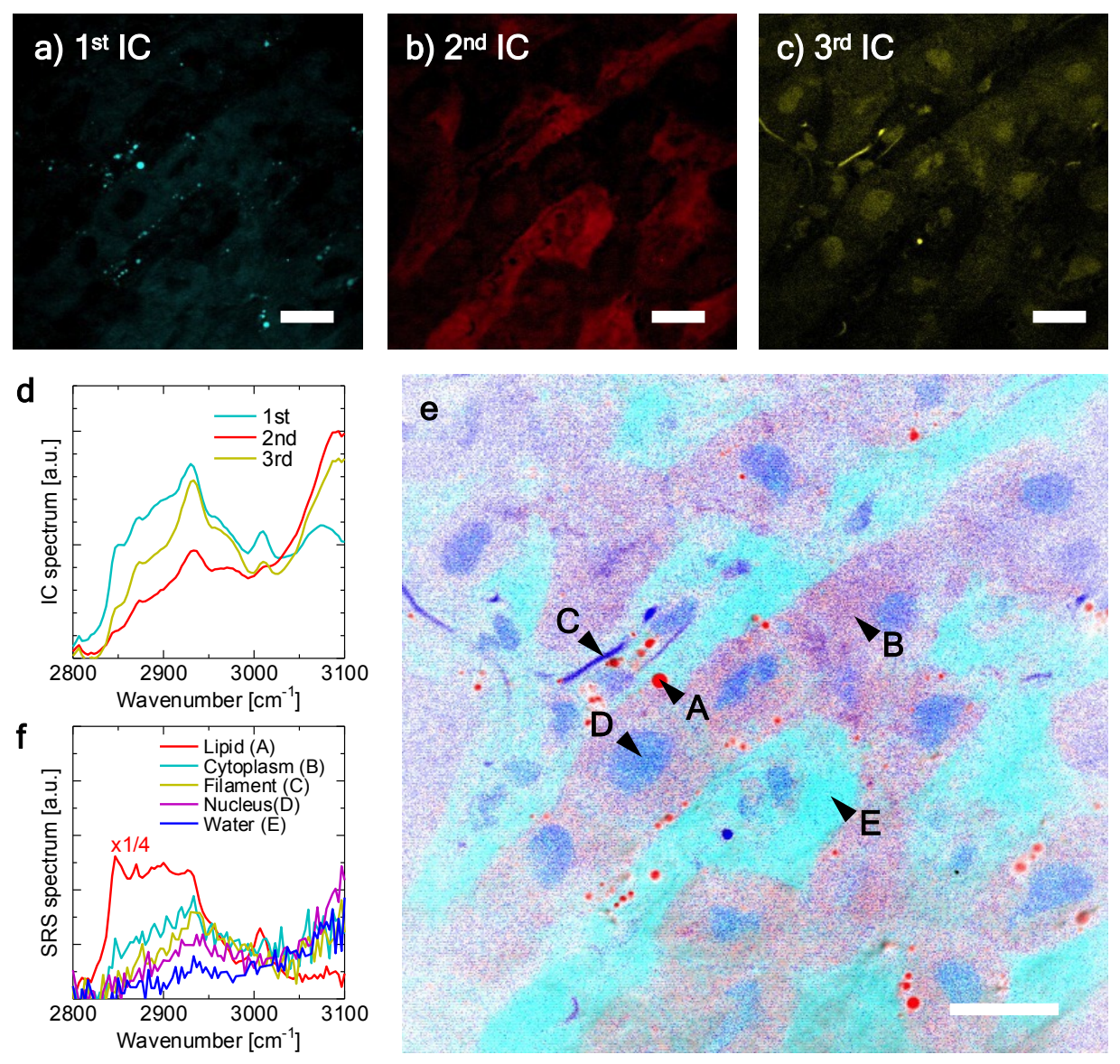

Fig. 4. Spectral imaging of a rat liver tissue [23]. 91 images at wavenumbers from 2800 to $3100 \mathrm{~cm}^{-1}$ were taken and averaged over 10 times. The total acquisition time was $<30 \mathrm{~s}$. The spectral images were analyzed by using 5 ICs. a. $1^{\text {st }}$ IC image reflecting the distribution of lipid-rich region. b. $2^{\text {nd }}$ IC image reflecting the distribution of water-rich regions. c. $3^{\text {rd }} \mathrm{IC}$ image reflecting the distribution of protein-rich region. d. IC spectra. e. Multicolor image produced by combining images a-c and inverting the contrast. A-E are explained in the text. f. SRS spectra in locations indicated by arrows in e. Scale bar: $20 \mu \mathrm{m}$.

We can further shorten the acquisition time when we observe only lipid droplets and fibrous structures, which produce strong SRS signals. Figure 5 demonstrates 3D observation of a vessel in the rat liver [23]. Taking advantage of the fast wavelength scanning capability, SRS images at 2850 and $2950 \mathrm{~cm}^{-1}$ were successively acquired while the $z$ position of the sample was scanned over 80 $\mu \mathrm{m}$ with a step of $1 \mu \mathrm{m}$. As a result, two-color SRS images with $500 \times 480 \times 81$ pixels were taken only in $<6 \mathrm{~s}$ (Figs. 5ab). The two-color images were analyzed by ICA. The $1^{\text {st }}$ and $2^{\text {nd }}$ IC images reflect the distributions of lipid droplets and fibrous texture, respectively (Figs. 5cd). This is consistent with the IC spectra shown in Fig. 5e, where the $1^{\text {st }}$ IC has both $\mathrm{CH}_{2}$ and $\mathrm{CH}_{3}$ stretching vibrations in 2850 and $2950 \mathrm{~cm}^{-1}$, respectively, and the $2^{\text {nd }} \mathrm{IC}$ has only $\mathrm{CH}_{3}$ stretching vibrations in 
$2950 \mathrm{~cm}^{-1}$. The entire images can be used to reconstruct a 3D image, with which we can see 3D structure of fibrous texture combined to the vessel (Fig. 5f). Such structural information would be useful for the research and diagnosis of cirrhosis of the liver and vascular lesion.

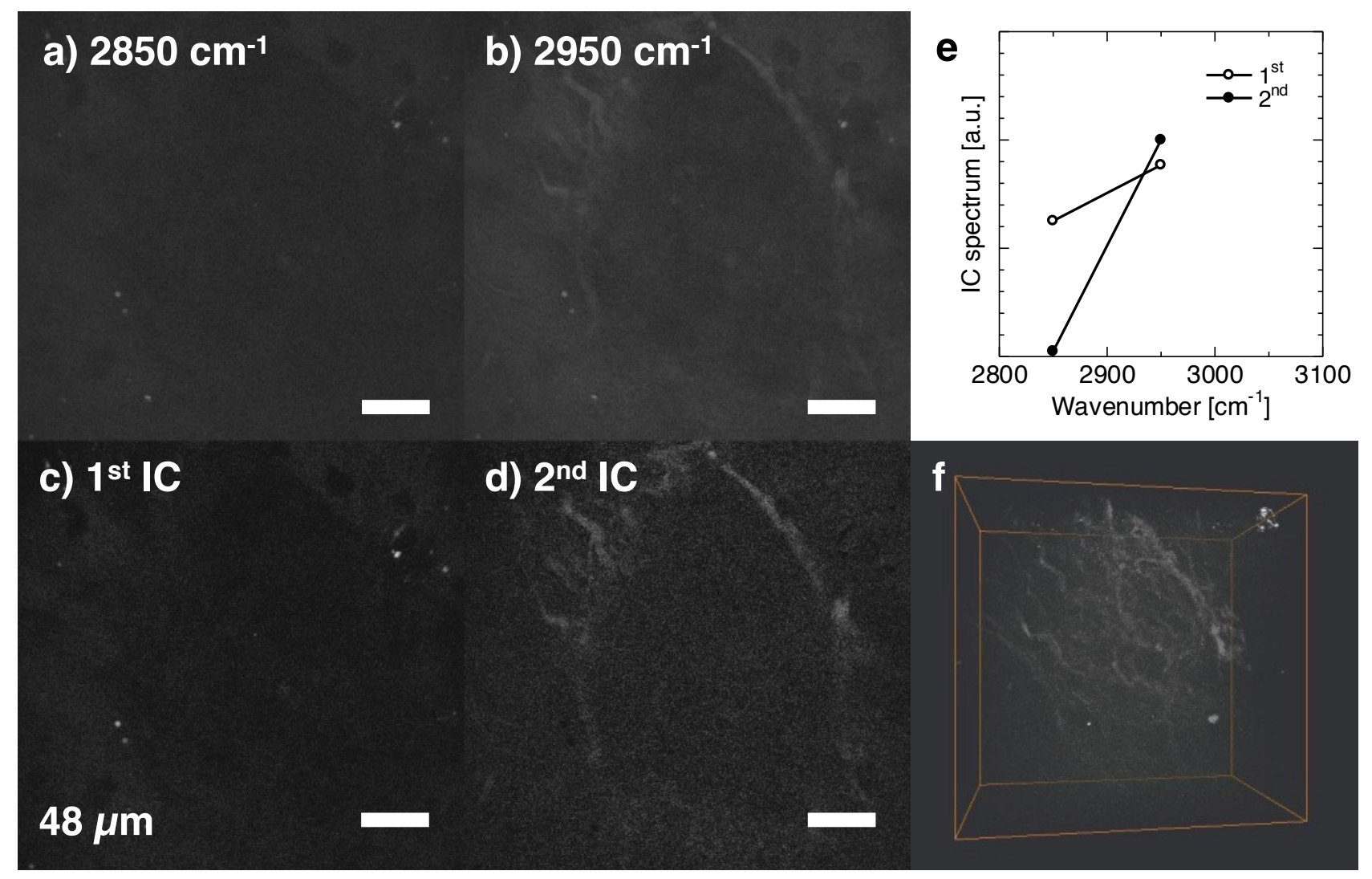

Fig. 5. 3D, two-color imaging of a vessel in the rat liver [23]. Images at wavenumbers of 2850 and $2950 \mathrm{~cm}^{-1}$ were successively taken while the $z$-position was scanned over $80 \mu \mathrm{m}$ with a step of $1 \mu \mathrm{m}$. $z=0$ corresponds to the deepest plane from the focusing lens. The total acquisition time was $5.3 \mathrm{~s}$. ad. Sectioned images at a position of $z=48 \mu \mathrm{m}$. a. SRS image at $2850 \mathrm{~cm}^{-1}$. b. SRS image at 2950 $\mathrm{cm}^{-1}$. c. $1^{\text {st }}$ IC image reflecting the distribution of lipid droplets. d. $2^{\text {nd }}$ IC image reflecting the distribution of fibrous texture. e. IC spectra. f. Reconstructed 3D image. Scale bar: $20 \mu \mathrm{m}$. 


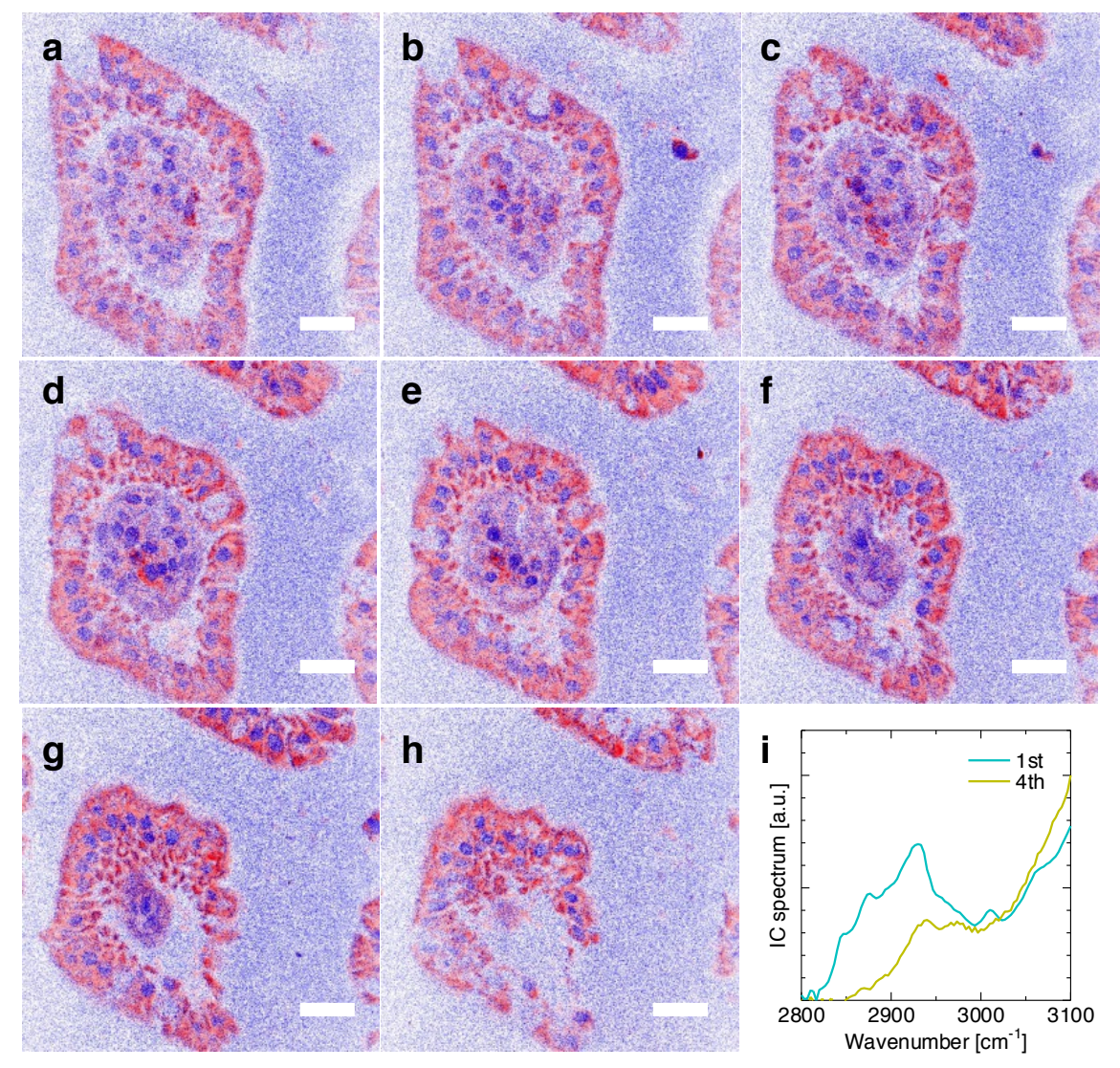

Fig. 6. Sectioned spectral imaging of intestinal villi in the mouse [23]. 91 images at wavenumbers from 2800 to $3100 \mathrm{~cm}^{-1}$ were taken by changing the $z$ position by $5.6 \mu \mathrm{m}$. The total acquisition time was $24 \mathrm{~s}$. The spectral images were analyzed by using 4 ICs. The $1^{\text {st }}$ IC (cytoplasm) and the $4^{\text {th }}$ IC (nuclei) images were colored by cyan and yellow, respectively, and then combined and the contrast was inverted. a-h. Sectioned multicolor images. f. Spectra of the $1^{\text {st }}$ and $4^{\text {th }}$ IC's. Scale bar: $20 \mu \mathrm{m}$.

It is also possible to conduct 3D sectioning in SRS spectral imaging. Figure 6 shows the 8 sections of multicolor images of intestinal villi in the mouse at different $z$ positions separated by $5.6 \mu \mathrm{m}$. For each section, 91 spectral images at wavenumbers from 2800 to $3100 \mathrm{~cm}^{-1}$ were taken. The total acquisition time was $24 \mathrm{sec}$. Figure $6 \mathrm{i}$ indicates that the $1^{\text {st }} \mathrm{IC}$ has large amount of $\mathrm{CH}_{2}$ stretching vibrations at $2850 \mathrm{~cm}^{-1}$, and the $4^{\text {th }} \mathrm{IC}$ is a mixture of $\mathrm{CH}_{3}$ stretching mode at $2930 \mathrm{~cm}^{-1}$ and the tails of $\mathrm{OH}$ stretching modes. Multicolor images shown in Figs. 6a-h are produced by combining the $1^{\text {st }}$ (cytoplasm, cyan) and $4^{\text {th }}$ (nuclei, yellow) IC images and inverting the contrast. We can clearly see the morphology of the tissue such as cell nuclei and cytoplasm. Note that, based on the conventional staining procedure, several thin slices are prepared, stained, and separately observed with a microscope. In contrast, our technique enables quick, label-free observation of unstained sample with sectioning capability. This would be powerful for medical diagnosis of tissues.

\section{CONCLUSION}

To summarize, we have demonstrated the label-free imaging of tissue based on the high-speed SRS spectral microscopy and the modified ICA. The developed system allows quick image acquisition at 
$>30$ frames/s by changing the wavenumber in a frame-by-frame manner. The modified ICA enables blind separation of constituents from SRS spectral images. The IC spectra give spectroscopic information, and the IC images can be used to produce multicolor images. The label-free, quick observation would be especially useful in the medical imaging of organs, and the implementation of the presented technique in SRS endoscopy [28, 29] would be straightforward and be advantageous for the application to in vivo imaging.

\section{REFERENCES}

[1] C. W. Freudiger, W. Min, B. G. Saar, S. Lu, G. R. Holtom, C. He, J. C. Tsai, J. X. Kang, and X. S. Xie, "Labelfree biomedical imaging with high sensitivity by stimulated Raman scattering microscopy," Science 322, 18571861 (2008).

[2] P. Nandakumar, A. Kovalev, and A. Volkmer, "Vibrational imaging based on stimulated Raman scattering microscopy," N. J. Phys. 11, 033026 (2009).

[3] Y. Ozeki, F. Dake, S. Kajiyama, K. Fukui, and K. Itoh, "Analysis and experimental assessment of the sensitivity of stimulated Raman scattering microscopy," Opt. Express 17, 3651-3658 (2009).

[4] B. G. Saar, C. W. Freudiger, J. Reichman, C. M. Stanley, G. R. Holtom, and X. S. Xie, "Video-rate molecular imaging in vivo with stimulated Raman scattering," Science 330, 1368-1370 (2010).

[5] M. N. Slipchenko, T. T. Le, H. Chen, and J. -X. Cheng, "High-speed vibrational imaging and spectral analysis of lipid bodies by compound Raman microscopy," J. Phys. Chem. B 113, 7681-7686 (2009).

[6] M. N. Slipchenko, H. Chen, D. R. Ely, Y. Jung, M. T. Carvajal and J. -X. Cheng, "Vibrational imaging of tablets by epi-detected stimulated Raman scattering microscopy," Analyst 135, 2613-2619 (2010).

[7] B. G. Saar, Y. Zeng, C. W. Freudiger, Y. -S. Liu, M. E. Himmel, X. S. Xie, and S. -Y. Ding, "Label-free, realtime monitoring of biomass processing with stimulated Raman scattering microscopy," Angew. Chem. Int. Ed. 122, 5608-5611 (2010).

[8] M. C. Wang, W. Min, C. W. Freudiger, G. Ruvkun, and X. S. Xie, "RNAi screening for fat regulatory genes with SRS microscopy," Nature Meth. 8, 135-138 (2011).

[9] B. G. Saar, L. R. Contreras-Rojas, X. S. Xie, and R. H. Guy, "Imaging drug delivery to skin with stimulated Raman scattering microscopy,” Mol. Pharmaceutics 8, 969-975 (2011).

[10] M. B. J. Roeffaers, X. Zhang, C. W. Freudiger, B. G. Saar, M. van Ruijven, G. van Dalen, C. Xiao, and X. S. Xie, "Label-free imaging of biomolecules in food products using stimulated Raman microscopy," J. Biomed. Opt. 16, 021118 (2011).

[11] X. Zhang, M. B. J. Roeffaers, S. Basu, J. R. Daniele, D. Fu, C. W. Freudiger, G. R. Holtom, X. S. Xie, "Labelfree live-cell imaging of nucleic acids using stimulated Raman scattering microscopy," ChemPhysChem. 13, 1054-1059 (2012).

[12] C. W. Freudiger, R. Pfannl, D. A. Orringer, B. G. Saar, M. Ji, Q. Zeng, L. Ottoboni, W. Ying, C. Waeber, J. R. Sims, P. L. De Jager, O. Sagher, M. A. Philbert, X. Xu, S. Kesari, X. S. Xie, G. S. Young, "Multicolored stain-free histopathology with coherent Raman imaging," Lab. Invest. 92, 1492-1502 (2012).

[13] Z. Yu, T. Chen, X. Zhang, D. Fu, X. Liao, J. Shen, X. Liu, B. Zhang, X. S. Xie, X.-D. Su, J. Chen and Y. Huang, "Label-free chemical imaging in vivo: three-dimensional non-invasive microscopic observation of amphioxus notochord through stimulated Raman scattering (SRS)," Chem. Sci. 3, 2646-2654 (2012).

[14] C. W. Freudiger, W. Min, G. R. Holtom, B. Xu, M. Dantus, and X. S. Xie, "Highly specific label-free molecular imaging with spectrally tailored excitation-stimulated Raman scattering (STE-SRS) microscopy," Nature Photon. 5, 103-109 (2011).

[15] D. Fu, F.-K. Lu, X. Zhang, C. Freudiger, D. R. Pernik, G. Holtom, and X. S. Xie, "Quantitative chemical imaging with multiplex stimulated Raman scattering Microscopy,” J. Am. Chem. Soc. 134, 3623-3626 (2012).

[16] F.-K. Lu, M. Ji, D. Fu, X. Ni, C. W. Freudiger, G. R. Holtom, X. S. Xie, "Multicolor stimulated Raman scattering microscopy," Mol. Phys. 110, 1927-1932 (2012).

[17] E. Ploetz, S. Laimgruber, S. Berner, W. Zinth and P. Gilch, "Femtosecond stimulated Raman microscopy," Appl. Phys. B 87, 389-393 (2007).

[18] E. R. Andresen, P. Berto, and H. Rigneault, "Stimulated Raman scattering microscopy by spectral focusing and fiber-generated soliton as Stokes pulse," Opt. Lett. 36, 2387-2389 (2011). 
[19] H. T. Beier, G. D. Noojin, and B. A. Rockwell, "Stimulated Raman scattering using a single femtosecond oscillator with flexibility for imaging and spectral applications," Opt. Express 19, 18885-18892 (2011).

[20] D. Fu, F.-K. F. Lu, X. Zhang, G. R. Holtom, C. W. Freudiger, M. Ji, X. Ni, X. S. Xie, "From coherent Raman microscopy to quantitative coherent Raman spectral imaging," Photonics West 2012, paper 8226-58 (2012).

[21] Y. Ozeki, W. Umemura, K. Sumimura, N. Nishizawa, K. Fukui, and K. Itoh, "Stimulated Raman hyperspectral imaging based on spectral filtering of broadband fiber laser pulses," Opt. Lett. 37, 431-433 (2011).

[22] J. L. Suhalim, J. C. Boik, B. J. Tromberg, and E. O. Potma, "The need for speed," J. Biophoton., 5, 387-395 (2012).

[23] Y. Ozeki, W. Umemura, Y. Otsuka, S. Satoh, H. Hashimoto, K. Sumimura, N. Nishizawa, K. Fukui and K. Itoh, "High-speed molecular spectral imaging of tissue with stimulated Raman scattering," Nature Photon. 6, 845-851 (2012).

[24] Y. Ozeki, Y. Kitagawa, K. Sumimura, N. Nishizawa, W. Umemura, S. Kajiyama, K. Fukui, and K. Itoh, "Stimulated Raman scattering microscope with shot noise limited sensitivity using subharmonically synchronized laser pulses," Opt. Express 18, 13708-13719 (2010).

[25] W. Umemura, K. Fujita, Y. Ozeki, K. Goto, K. Sumimura, N. Nishizawa, K. Fukui, and K. Itoh, "Subharmonic synchronization of picosecond $\mathrm{Yb}$ fiber laser to picosecond Ti:Sapphire laser for stimulated Raman scattering microscopy,” Jpn. J. App. Phys. 51, 022702 (2012).

[26] A. Hyvärinen, J. Karhunen, and E. Oja, Independent component analysis, Wiley, New York, 2001.

[27] V. Vrabie, C. Gobinet, O. Piot, A. Tfayli, P. Bernard, R. Huez, and M. Manfait, "Independent component analysis of Raman spectra: Application on paraffin-embedded skin biopsies," Biomed. Signal Process. Control 2, 40-50 (2007).

[28] S. Brustlein, P. Berto, R. Hostein, P. Ferrand, C. Billaudeau, D. Marguet, A. Muir, J. Knight, and H. Rigneault, "Double-clad hollow core photonic crystal fiber for coherent Raman endoscope," Opt. Express 19, 1256212568 (2011).

[29] B. G. Saar, R. S. Johnston, C. W. Freudiger, X. S. Xie, and E. J. Seibel, "Coherent Raman scanning fiber endoscopy," Opt. Lett. 36, 2396-2398 (2011). 